\title{
Mechanical Behavior of a Series of Copolyester Blends near the Glass Transition: Monotonic and Load-Hold Behavior in Compression
}

\author{
Gurucharan Chandrasekaran and Rebecca B. Dupaix \\ Mechanical and Aerospace Engineering Department, The Ohio State University, Columbus, OH 43210, USA \\ Correspondence should be addressed to Rebecca B. Dupaix, dupaix.1@osu.edu
}

Received 22 June 2011; Revised 9 September 2011; Accepted 3 October 2011

Academic Editor: Wen Fu Lee

Copyright ( 2012 G. Chandrasekaran and R. B. Dupaix. This is an open access article distributed under the Creative Commons Attribution License, which permits unrestricted use, distribution, and reproduction in any medium, provided the original work is properly cited.

\begin{abstract}
Monotonic loading tests were conducted on five commercial blends of poly(ethylene terephthalate) (PET) and poly(1,4cyclohexylenedimethylene terephthalate) (PCT) at temperatures of $90^{\circ} \mathrm{C}$ and $100^{\circ} \mathrm{C}$ and strain rates of $0.1 / \mathrm{s}, 0.05 / \mathrm{s}$, and $0.005 / \mathrm{s}$ in uniaxial and plane strain compression. On comparing the mechanical behavior of the five materials, it was found that the behavior of the low-PCT content materials was different from the high-PCT content materials only at conditions that favored strain-induced crystallization, particularly in plane strain compression. Load-hold tests were also conducted on three of the blends with similar results to the monotonic tests. Material differences were only pronounced at certain conditions, and in these cases the low-PCT content materials showed increased strain hardening after the hold period while the high-PCT content material did not. Therefore, it was found that the addition of a hold period was not exclusively required to observe differences in the crystallizable materials over the noncrystallizing blends. The increased strain hardening likely associated with crystallization in PET was only observed when the following conditions were met: (i) strain rates of $0.1 / \mathrm{s}$ and above, (ii) temperatures of $90^{\circ} \mathrm{C}-100^{\circ} \mathrm{C}$, (iii) plane strain compression, and (iv) after a certain level of deformation.
\end{abstract}

\section{Introduction}

Poly(ethylene terephthalate) (PET) is a thermoplastic polymer that is extensively used in applications such as drawn fibers, bottles, and stretched films. PET has the ability to crystallize when deformed at temperatures slightly above the glass transition $\left(\mathrm{T}_{\mathrm{g}}\right)$ by a phenomenon called stressor strain-induced crystallization. When it undergoes straininduced crystallization, the density, stiffness, resistance to gas permeability, and dimensional stability of the material increase without affecting its optical transparency, because of the very small (nanoscale) size of the crystallites. As a result, PET is frequently processed in the range of 10 to $20^{\circ} \mathrm{C}$ above $\mathrm{T}_{\mathrm{g}}$. The crystallization process and the mechanical behavior of PET above $T_{g}$ are highly dependent on temperature, strain rate, and the mode of deformation. It is difficult to experimentally isolate these dependencies and determine their effects, particularly for complex $3 \mathrm{D}$ processes, and the need exists for reliable material models that can be used in finite element simulations in this temperature regime. Any potential model must capture the high temperature, strain rate, and strain state-dependent behavior of PET. In addition to these dependencies, the material model should also incorporate the effect of strain-induced crystallization to be able to effectively simulate a manufacturing process that involves PET.

Previous work has largely involved conducting experiments at simple monotonic load conditions. Also, the issue of occurrence of strain-induced crystallization and its effect on the strain-hardening behavior of PET at large strains continues to be unclear. One previous study attempted to isolate effects of strain-induced crystallization by comparing PET3.5 (3.5\% PCT) and PETG (31\% PCT) [1]. This work looks to build on that previous research and address these open issues by (i) conducting monotonic tests to carry out a comparative study of the mechanical behavior of five different PET-PCT 
blends and (ii) conducting tests with nonmonotonic load histories to gain a better understanding of the factors that influence the occurrence and effects of crystallization.

\section{Background}

2.1. Experimental Background. Various experimentalists have studied the effects of strain-induced crystallization on the behavior of PET through mechanical and thermal characterization. Mechanical characterization has included uniaxial tension, biaxial tension, uniaxial compression, and plane strain compression tests. Thermal characterization has included the use of differential scanning calorimetry (DSC) to estimate the crystallinity content as a function of temperature, strain rate, strain state, and final strain. Wide-angle X-ray diffraction (WAXD) measurements are also used to determine the nature of the evolved crystallinity and the development of crystal texture with temperature, strain rate, strain state, and final strain.

Zaroulis and Boyce [2] conducted compression tests and DSC analysis on initially amorphous PET to determine the contribution of molecular orientation versus strain-induced crystallization to the significant strain-hardening behavior of PET observed at large strains. These tests were conducted up to $\mathrm{T}_{\mathrm{g}}$ over a wide range of strain rates. They inferred from the results that strain hardening was caused solely by orientation in uniaxial compression and by both orientation and crystallization in plane strain compression. Llana and Boyce [3] added to this experimental work by performing mechanical tests and microstructural analyses on PET above $\mathrm{T}_{\mathrm{g}}$. They observed from the mechanical tests, that the stressstrain behavior of PET mainly consisted of four distinct features: (1) a relatively high initial stiffness, (2) a rollover to flow rather than a distinctive yield stress, (3) a moderate strain hardening with increasing strain, and (4) dramatic strain hardening at large strains. From the DSC results they found that the crystallinity content in PET increases with decreasing temperature and increasing strain rate. Using WAXD results, they also found the evolution of the crystallographic texture in both the strain states to be consistent with the molecular orientation of the respective strain state. Therefore, these tests established the fact that both the mechanical behavior and the structural evolution of PET depend on temperature, strain rate, and strain state above the glass transition temperature.

Complicating the ability to directly correlate mechanical experimental conditions with postexperimentally measured crystallinity is the practical difficulty of quenching a sample before crystallization occurs due to the brief annealing period at the end of deformation. As a result, it is difficult to determine whether crystallization occurs during or after deformation. Blundell et al. [4] used in situ experiments in which X-ray patterns were recorded during the deformation process to gain a better understanding of the occurrence and effects of crystallization during the large strain deformation of PET. By conducting tensile tests over a wide range of rates $(0.05-12 / \mathrm{s})$, they found that there are three regimes of PET deformation. (1) At draw rates faster than the rate of chain retraction, crystallization is delayed until the end of draw. (2) At draw rates that are slower than the chain reptation rate, no crystallization occurs. (3) For draw rates between the two above-mentioned rates, crystallization can start before the end of draw. Other important conclusions drawn from their work were that both orientation and crystallization occur only within a certain range of temperature and draw ratio and the presence of oriented noncrystalline polymer segments is essential for crystallization to occur.

Work by Dupaix and Boyce [1] looked at comparing the stress-strain behavior of PET with PETG, which is a noncrystallizing copolymer of PET. The comparison in behavior was done across a wide range of temperatures (25$110^{\circ} \mathrm{C}$ ) and strain rates $(.005-1.0 / \mathrm{s})$ in both uniaxial and plane strain compression. Their results clearly indicated that at most of the conditions, the mechanical behavior of PET and PETG was similar and that they exhibit similar trends with respect to temperature, strain rate, and strain state, even though PET is a crystallizing polymer and PETG is not. This led to the suggestion that the dramatic hardening that occurs at large compressive strains in PET is mainly caused by increased molecular orientation and not necessarily by strain-induced crystallization.

2.2. Constitutive Modeling Background. Due to its wide industrial use, various research groups have tried to model the behavior of PET under deformation near the glass transition temperature. Several of the key models are summarized here.

Buckley et al. [5] created a physically based three-dimensional model to capture the behavior of amorphous polymers near the glass transition. This model was formulated on the idea that the total stress on deformation would be the sum of the individual stress components arising from bond stretching and conformational change. For the bond distortion part, the model used linear elasticity and Eyring viscous flow; for the conformation part, the model used the Edwards-Vilgis entropy function.

Boyce et al. [6] proposed a constitutive model for PET that captured its behavior above $T_{g}$ across a wide range of strain rates in uniaxial and plane strain compression. In this model the total resistance offered by the material to deformation is broken down into (1) the resistance that exists between the polymer molecules due to secondary bonding, and (2) the network resistance that occurs due to the underlying network of polymer chains. Crystallization, which was considered to provide an additional strain-hardening mechanism, was incorporated through increasing the shear resistance after a critical network stretch was reached.

Another model was proposed by Vigny et al. [7] that incorporates several of the same features: thermally activated viscoplastic flow, the eight-chain model for orientation and hardening at large strains, and elements that stiffen with the evolution of crystallinity.

Dupaix and Boyce $[8,9]$ built on the Boyce model [6] to capture the strong dependence of the stress-strain behavior of the noncrystallizing PETG on temperature, strain rate, and strain state, from below to above $T_{g}$ and up to very large strains. A physical parameter which is independent of 
temperature and strain rate, called the orientation parameter, was used as the governing variable for molecular relaxation and especially for its cessation in that work.

Work by Ahzi and coworkers [10, 11] has greatly advanced the modeling of strain-induced crystallization in PET through a series of recent papers. Their earlier model built on the Boyce model [6], adding a separate branch to account for the evolving semicrystalline phase in both an upper bound and lower bound configuration [10]. Later, they extended this to a self-consistent model [11]. In this work, they used a model by Flory [12] to predict the onset of crystallization as a function of temperature and the stretch in the material.

Dupaix and Krishnan [13] formulated a hybrid model to account for the behavior of PET and PETG under both simple and complex (load-hold) conditions. This hybrid model was the model of Dupaix and Boyce modified to include strain-induced crystallization following the upper bound model of Ahzi. In addition, criteria to delay the onset of strain-induced crystallization were included. The criteria restricted strain-induced crystallization to occur only (1) after a particular level of deformation has been reached and (2) after the strain rate has slowed below a specific rate. However, no load-hold experimental data was available to compare with the simulation results obtained from that work.

\section{Experimental Methods}

3.1. Material. The five different blends of poly(ethylene terephthalate) (PET) and poly(1,4-cyclohexylenedimethylene terephthalate) (PCT) used in these experiments were supplied by Eastman Chemical Co. as $10 \mathrm{~cm} \times 10 \mathrm{~cm} \times 3.2 \mathrm{~mm}$ injection molded plaques. The composition of the five blends is PET00 (0\% PCT), PET1.5 (1.5\% PCT), PET3.5 (3.5\% PCT), PET12 (12.5\% PCT), and PETG (31\% PCT). The materials are identical to those used in Chen et al. [14]. The glass transition temperature of these materials is approximately $77^{\circ} \mathrm{C}$ and there is very little variation in glass transition temperature among the materials. In addition the materials as received were optically clear and amorphous. While some small initial anisotropy arising from the fabrication of the plaques is unavoidable, we expect this to be fairly small and we avoided taking samples from areas such as near the edges or the runners.

For uniaxial compression tests, cylindrical specimens with a diameter of $12.3 \mathrm{~mm}$ and a height to diameter ratio of 0.2 were used. For plane strain compression tests, rectangular samples with a width and length of $10 \mathrm{~mm}$ and a height of $3.2 \mathrm{~mm}$ were used. Prior to testing, the specimens were stored in a desiccant chamber.

3.2. Experimental Setup and Method. An Instron 5869 electromechanical test frame was used to carry out the experiments. A $50 \mathrm{KN}$ load cell recorded force data while an LVDT measured the distance between the compression platens during testing. The crosshead speed was controlled using a personal computer with Windows XP and Instron Bluehill software. The speed was constantly decreased throughout the course of the experiment in order to maintain a constant true strain rate in compression.

Temperature was controlled using an environmental chamber that was heated to the required temperature for one hour prior to any testing to obtain a uniform temperature distribution within the test chamber. Teflon sheets were placed between the compression platens and the specimen and also between the specimen and the channel die (in the case of plane strain) with a light amount of WD-40 lubricant between the Teflon and the steel fixtures in order to minimize the effects of friction. Care was taken not to expose the specimen to the lubricant. Each test sample was heated in the chamber for 20 minutes to ensure uniform sample temperature. Care was taken to ensure that all the test samples were subjected to the same thermal history.

\section{Experimental Results and Discussions}

4.1. Monotonic Tests. Monotonic tests were conducted at test temperatures of $90^{\circ} \mathrm{C}$ and $100^{\circ} \mathrm{C}$ and at strain rates of $0.1 / \mathrm{s}$, $0.05 / \mathrm{s}$, and $0.005 / \mathrm{s}$ in both uniaxial compression and plane strain compression for all five PET-PCT blends.

In compression, it is observed that all five materials exhibit the four distinct features of the stress strain curve that have been observed by previous researchers for PET3.5 and PETG above the glass transition temperature. These features include (1) a relatively high initial stiffness, (2) a rollover to flow rather than a distinct yield stress, (3) moderate strain hardening with increasing strain, and (4) dramatic strain hardening at large strains. Each of these features was seen to depend on both strain rate and temperature for all five materials.

Figure 1 shows a representative plot of uniaxial compression stress-strain data of the five materials. This plot is for $90^{\circ} \mathrm{C}$ and a strain rate of $0.1 / \mathrm{s}$, with data at other temperatures and strain rates being very similar. Namely, in uniaxial compression, there is no significant difference in the stressstrain behavior of these materials, other than the fact that PET12 tends to be the softest of the five materials. Otherwise, any measured difference among the samples was similar to the difference measured between two samples of the same material.

Figure 2 shows the strain rate dependence of PET00 at a temperature of $90^{\circ} \mathrm{C}$ in uniaxial compression. Both the initial modulus and flow stress increase with increasing strain rate for all five materials. The subsequent strain hardening that occurs with increasing strain increases as the strain rate increases. The strain level at which the dramatic hardening occurs shifts to lower strains with increasing strain rate.

Figure 3 shows the temperature dependence of PET00 at a strain rate of $0.1 / \mathrm{s}$ in uniaxial compression. It was observed that the initial modulus, flow stress, and the subsequent strain hardening decrease with increasing temperature. Also, the dramatic strain hardening at large strains shifts to higher strains at higher temperatures. Though Figures 2 and 3 show only the temperature and strain rate dependence of PET00, the behavior of all five materials showed similar temperature and strain rate dependence. 


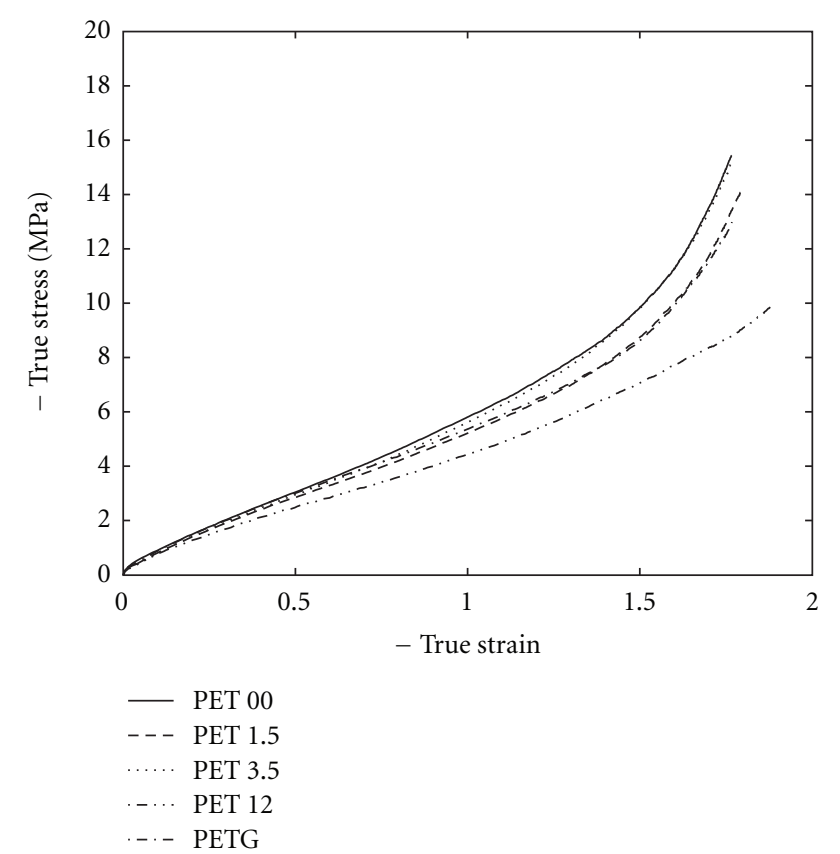

Figure 1: Material comparison, uniaxial compression at $90^{\circ} \mathrm{C}$ and $0.1 / \mathrm{s}$.

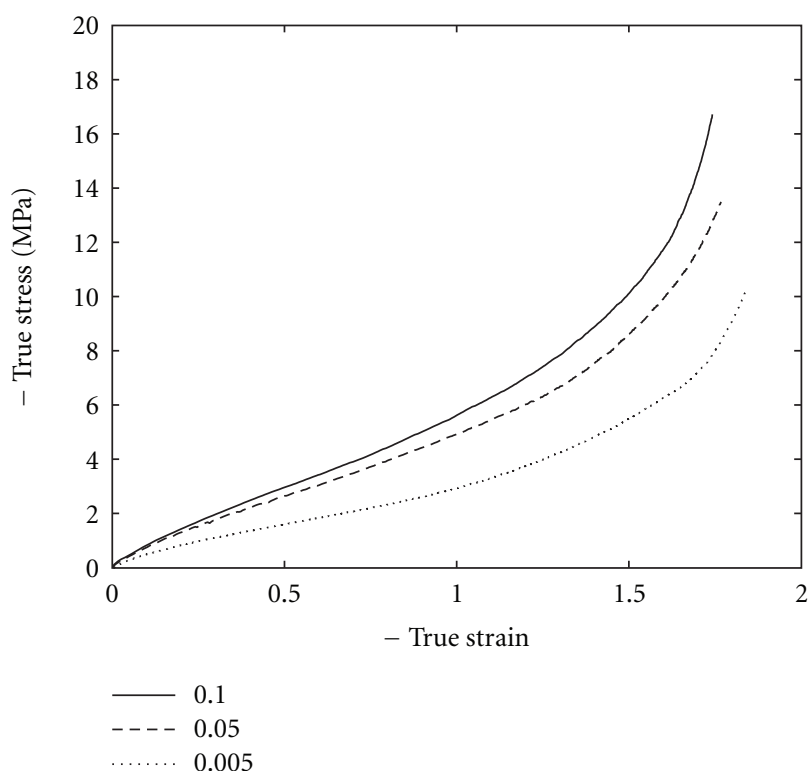

FIGURE 2: Strain rate comparison, PET00, and uniaxial compression at $90^{\circ} \mathrm{C}$.

In Figures 4-6, the stress strain behavior of the five materials in plane strain compression is compared. From these curves it was observed that for a strain rate of $0.1 / \mathrm{s}$ and temperatures of $90^{\circ} \mathrm{C}$ and $100^{\circ} \mathrm{C}$, PET00, PET1.5, and PET3.5 exhibited dramatic strain hardening at higher strains while PET12 and PETG did not. This could be attributed to the occurrence of strain-induced crystallization or a highly ordered mesophase in the three materials with low-PCT content. However, at the experimental condition of $90^{\circ} \mathrm{C}$ and $0.005 / \mathrm{s}$ (Figure 5), none of the materials exhibited any

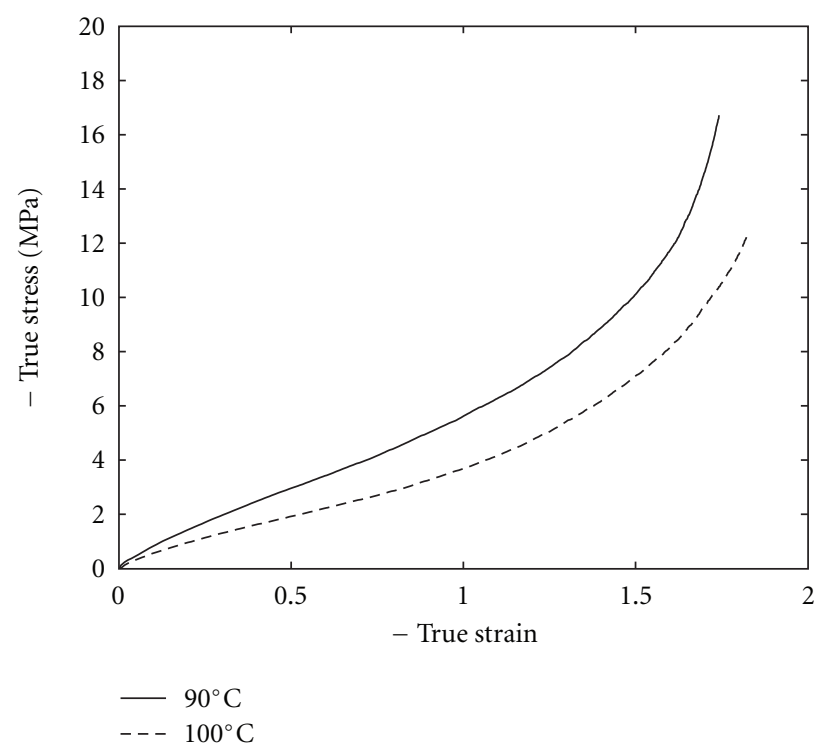

FIgURE 3: Temperature comparison, PET00, and uniaxial compression at $0.1 / \mathrm{s}$.

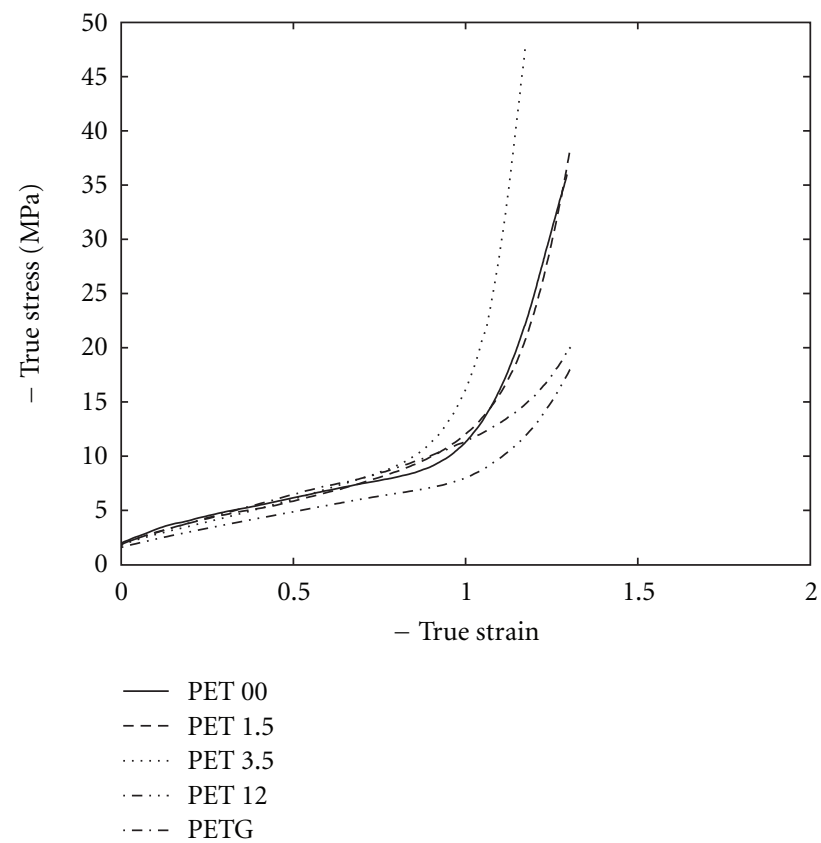

Figure 4: Material comparison, plane strain compression at $90^{\circ} \mathrm{C}$ and $0.1 / \mathrm{s}$.

strain hardening at higher strains. This could be explained by the fact that at a slower rate of $0.005 / \mathrm{s}$, molecular relaxation occurs more quickly, preventing the development of significant molecular orientation and also hindering straininduced crystallization.

Figure 7 shows the strain state dependence of the mechanical behavior of PET00 above the glass transition temperature $\left(90^{\circ} \mathrm{C}\right.$ and $\left.0.1 / \mathrm{s}\right)$. It was seen in these figures that the initial modulus, flow stress, and the subsequent strain hardening are much higher in plane strain compression 


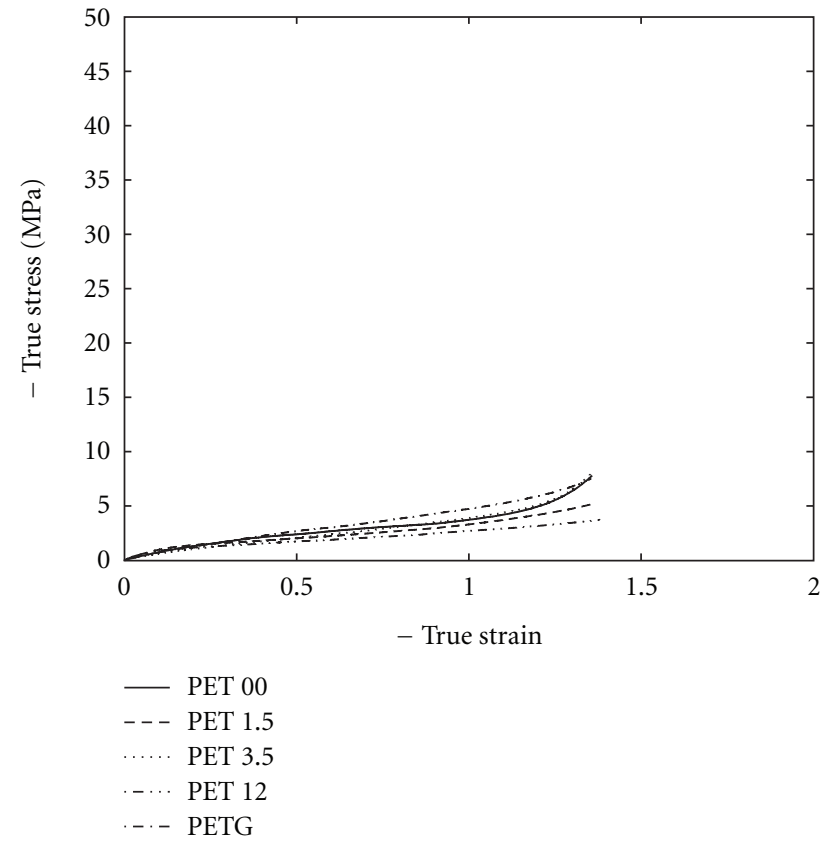

Figure 5: Material comparison, plane strain compression at $90^{\circ} \mathrm{C}$ and $0.005 / \mathrm{s}$.

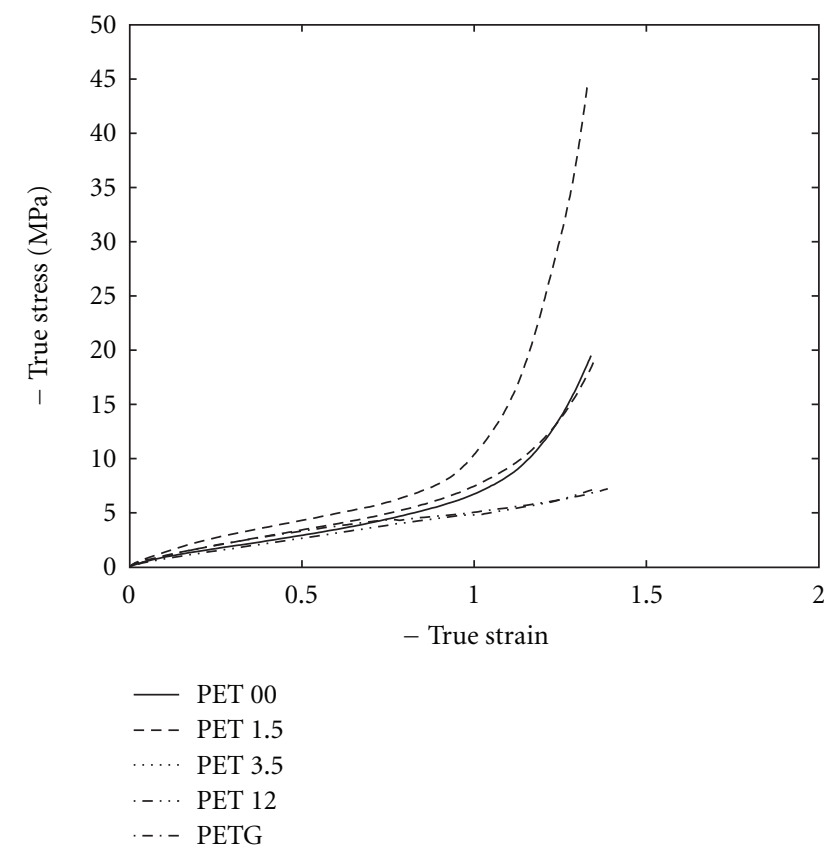

Figure 6: Material comparison, plane strain compression at $100^{\circ} \mathrm{C}$ and $0.1 / \mathrm{s}$.

when compared to uniaxial compression for the same temperature and strain rate. The dramatic hardening that occurs at high strains also occurs at a lower strain level in plane strain compression than in uniaxial compression. The same trend was seen with the other materials. We also observed that the stress-strain curves exhibit the same dependence on strain rate and temperature in plane strain compression as in uniaxial compression (data not shown).

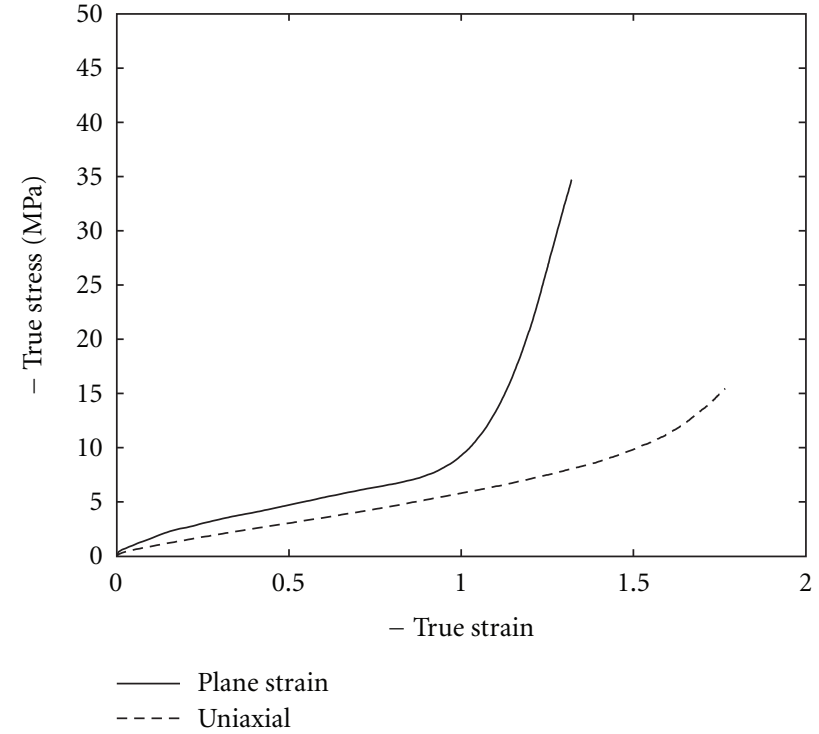

FIgURE 7: Strain state comparison, PET00, and $90^{\circ} \mathrm{C}$ and $0.1 / \mathrm{s}$.

To conclude, PET00, PET1.5, and PET12 exhibit the same dependence on strain rate, temperature, and strain state as PET3.5 and PETG (studied in detail in previous research by Dupaix and Boyce [1]). In plane strain compression, it was seen that PET00 and PET1.5 behaved in a similar manner to PET3.5 while PET 12 behaved more like PETG. This was expected as PET00, PET1.5, and PET3.5 have lower PCT content while PET12 and PETG have relatively higher PCT content. It should also be noted that Dupaix and Boyce [1] found very little difference between PET and PETG in uniaxial compression and only saw significant differences in plane strain compression. Therefore, the finding in this work that the five materials behave similarly in uniaxial compression and exhibit differences only in plane strain compression is consistent with those results.

4.2. Load-Hold Tests. Crystallization is favored in PET when it is deformed at strain rates that are fast enough for molecular orientation to develop. However, at higher strain rates, the polymer chains have limited time to crystallize during the deformation. Therefore, it is hypothesized that deforming PET at a high strain rate and subsequently holding the material at a constant strain level would provide adequate molecular orientation during deformation and would also allow enough time for crystallization to occur during the hold period. This would also mean that PET and PETG should exhibit different behavior in load-hold tests.

In uniaxial compression, load-hold tests were conducted at temperatures of $90^{\circ} \mathrm{C}$ and $100^{\circ} \mathrm{C}$ and at strain rates of $0.1 / \mathrm{s}$ and $0.005 / \mathrm{s}$. At each experimental condition, the deformation was paused and held at true strains of either -1.4 or -1.7. Plane strain compression tests were also conducted at the same strain rates and temperatures, but with the hold taking place at a true strain of -1.0 , which provides the same chain stretch as holding a sample at -1.4 in uniaxial compression (chain stretch refers to the stretch along the diagonal of a unit cube aligned with the principal axes 


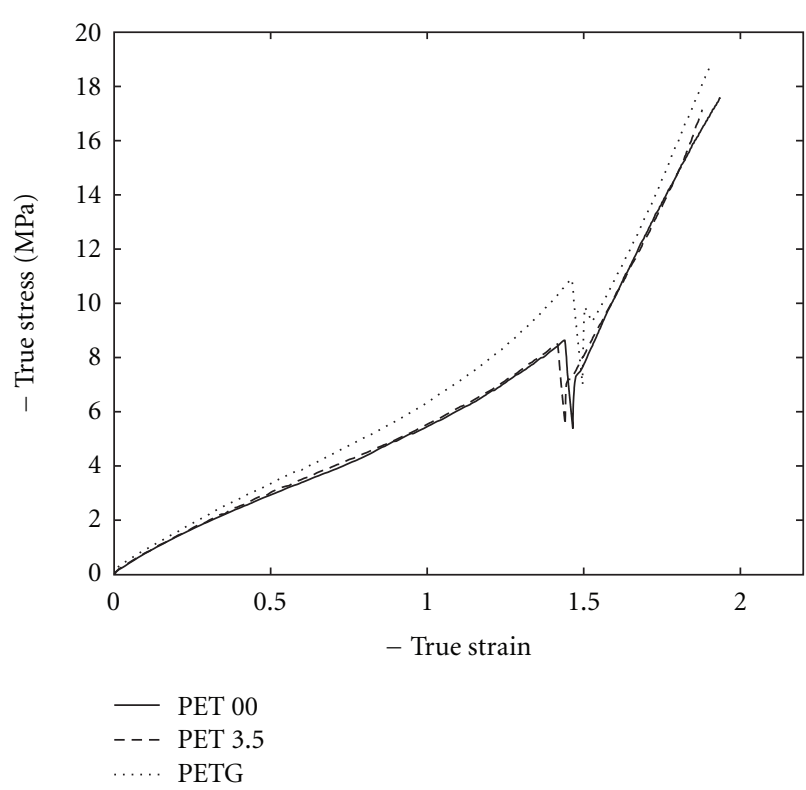

Figure 8: Material comparison, uniaxial compression at $90^{\circ} \mathrm{C}$ and $0.1 / \mathrm{s}$, and hold at -1.4 .

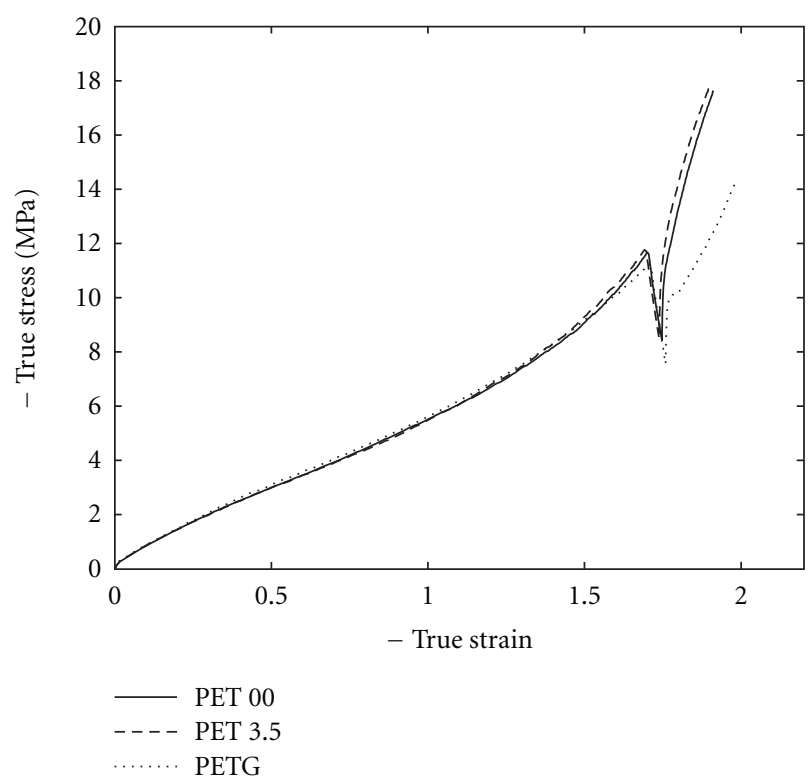

Figure 9: Material comparison, uniaxial compression at $90^{\circ} \mathrm{C}$ and $0.1 / \mathrm{s}$, and hold at -1.7 .

of deformation [15] and is used here as an effective stretch to better compare the amount of deformation a polymer sample would experience in two different deformation modes). Middleton et al. [16] observed that strain-induced crystallization occurs very rapidly after deformation $(<1 \mathrm{sec})$. In this work, a hold of 20 seconds was chosen, which should be more than sufficient to allow crystallization to initiate.

Figure 8 shows a representative plot of uniaxial stressstrain behavior of the three materials at $90^{\circ} \mathrm{C}$ and a strain rate of $0.1 / \mathrm{s}$, with a hold at a true strain of -1.4 . Qualitatively similar results were observed at the other strain rates and

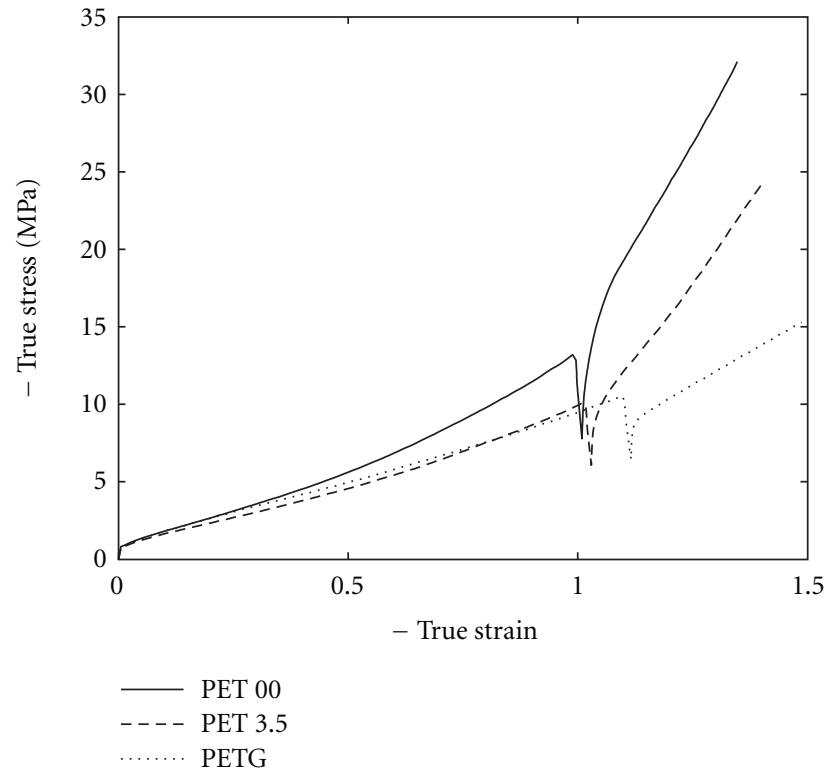

FIGURE 10: Material comparison, plane strain compression at $90^{\circ} \mathrm{C}$ and $0.1 / \mathrm{s}$, and hold at -1.0 .

temperature. Figure 9 shows the one exception in uniaxial compression. This figure shows data, also at $90^{\circ} \mathrm{C}$ and $0.1 / \mathrm{s}$, but with the hold delayed until -1.7 . In this one case, PET00 and PET3.5 show slightly increased hardening after the hold compared to PETG. Yet the difference does not seem significant enough to make a strong conclusion about the contribution of strain-induced crystallization.

In contrast, Figure 10 shows the stress strain behavior of the three materials in plane strain compression at $90^{\circ} \mathrm{C}$ and $0.1 / \mathrm{s}$ with a hold occurring at a true strain of -1.0 . From these curves it was observed that at this temperature and strain rate, PET00 and PET3.5 exhibited dramatic strain hardening at higher strains while PETG did not. This could be attributed to the occurrence of strain-induced crystallization in PET00 and PET3.5 under these favorable conditions. This effect was still present but less pronounced at the other temperature and rates that were tested.

The experimental results indicate that even with the addition of a hold period, most of the test conditions did not show significant material differences, with the exception of one or two specific test conditions. The differences were most evident in plane strain compression, a result that was already observed in the monotonic results.

The conclusions we can draw are somewhat limited, in the absence of real-time data on crystallinity. However, whether crystallization begins during deformation or only upon holding/annealing, it appears that in uniaxial compression, the ability of the material to crystallize has little effect on the stress-strain curve, with or without a hold period. This is further supported by the strong similarity in strain-hardening behavior across all of the copolyesters tested. In plane strain compression, the ability of the material to crystallize has much more of an effect on the large strain stiffening, a result that is not appreciably changed with the addition of a hold period, though the hold period seems 
to cause the strain hardening to increase even further at particular conditions. Since the hold period has only a small effect, it probably implies that crystallization had already begun during the deformation. In uniaxial compression, strain-induced crystallization does not significantly affect the stress-strain behavior, but in plane strain compression it appears to, likely because of the single flow direction in plane strain compression that leads to a high degree of crystal alignment with that flow direction [3].

\section{Crystallization Onset Criteria}

We propose here some possible crystallization onset criteria based on these experimental results.

Amount of Deformation. Previous work has established that the polymer chains attain a highly oriented state after a significant level of deformation is attained which helps crystallization to occur. To better define the strain level at which crystallization occurs, tests were conducted with holds at true strains of $-1.0,-0.9,-0.85$, and -0.7 . A temperature of $90^{\circ} \mathrm{C}$, strain rate of $-0.1 / \mathrm{s}$, and plane strain compression were used, since these conditions showed the most strain hardening in the earlier tests. From Figure 11, it can be seen that the additional strain hardening occurs for holds at -0.9 and -1.0 and not for holds at -0.85 and -0.7 . Therefore, it can be inferred that strain-induced crystallization first occurs for these experimental conditions at a true strain of -0.9 .

To correlate the observed strain level with a more physical representation of deformation level, we use the chain stretch as an equivalent strain measure:

$$
\lambda_{c h}=\frac{1}{\sqrt{3}} \sqrt{\lambda_{1}^{2}+\lambda_{2}^{2}+\lambda_{3}^{2}}
$$

where $\lambda_{1}, \lambda_{2}$, and $\lambda_{3}$ are the principal stretches.

The chain stretch corresponding to the true strain value of $\varepsilon=-0.9$ is determined to be $\lambda_{c h}=1.55$. Therefore, straininduced crystallization is taken to occur when the chain stretch reaches 1.55 .

\section{Condition 1: Crystallize if $\lambda_{c h}>1.55$.}

Mode of Deformation. Experimental results clearly showed that the additional strain hardening observed during large strain deformation of PET was predominantly present in plane strain compression (see Figures 8 and 10). This can be attributed to the occurrence of strain-induced crystallization which is favored by the unidirectional alignment of the polymer chains along the flow direction in plane strain compression. Therefore, this observation that crystallization occurs only in plane strain deformation should be incorporated into the constitutive model as an initiation condition. This is done using the fact that the principal stretches are different for the two different modes of deformation. In uniaxial compression, two principal stretches are greater than one (with the third principal stretch less than one). In plane strain compression, however, only one principal stretch is greater than one. Therefore, a criterion that prevents crystallization when two principal stretches are large will achieve

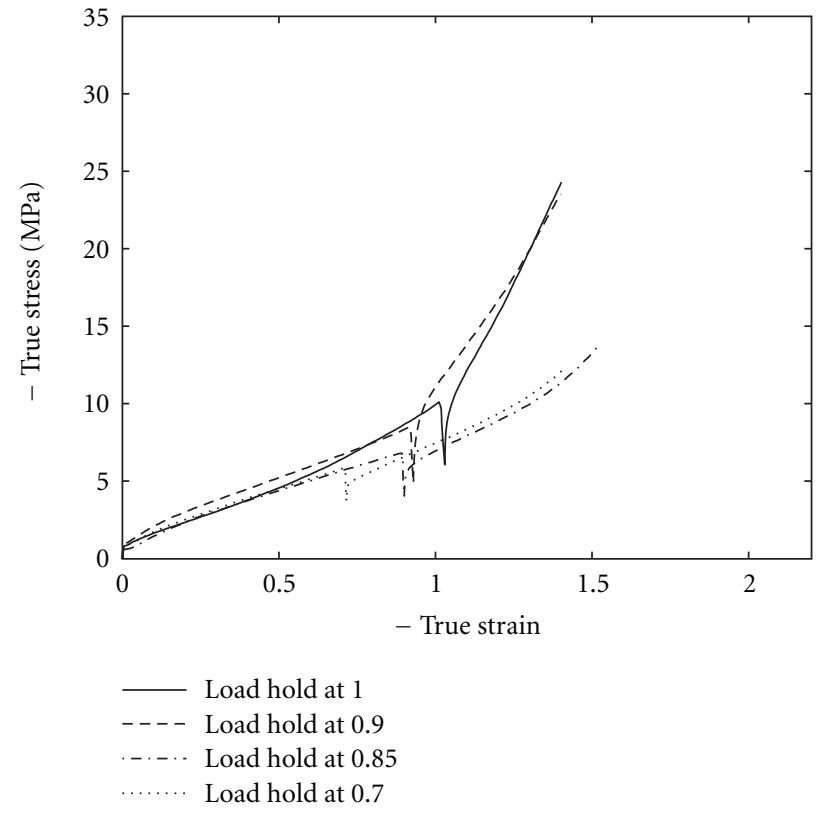

FIgURE 11: Stress-strain behavior of PET3.5 at $90^{\circ} \mathrm{C}$ and $0.1 / \mathrm{s}$, plane strain compression with different hold strains.

our goal of only allowing crystallization during plane strain compression. Here, we arbitrarily choose 1.3 to prevent crystallization during uniaxial compression simulations.

Condition 2: Do not crystallize if both $\lambda_{1}>1.3, \lambda_{2}>1.3$.

Strain Rate. Strain rate clearly influences the occurrence of crystallization. Dupaix and Krishnan [13] proposed that PET should undergo a certain amount of deformation and the deformation should slow to a very low rate $\left(\dot{\gamma}_{A}<\right.$ $0.009 / \mathrm{s}$ ) for strain-induced crystallization to occur during the deformation of PET. These conditions were based on the experimental investigations performed by Mahendrasingam et al. [17-19] and Middleton et al. [16].

Here, we observed that crystallization-caused strain hardening did not appear during testing at a strain rate of $0.005 / \mathrm{s}$ (lower than $0.009 / \mathrm{s}$ ). As a result, if we want to restrict crystallization to only occur during a hold period, the critical strain rate must be lower than this; here we will use $\dot{\gamma}_{A}<$ $0.0009 / \mathrm{s}$ as the criterion.

Condition 3: Crystallize if $\dot{\gamma}_{A}<0.0009 / \mathrm{s}$.

Alternate Criteria to Consider. On comparing the monotonic test results with the load-hold test results, it was seen that the experimental conditions at which strain hardening occurs in PET00 and PET3.5 are the same in the monotonic tests and load-hold tests. Strain hardening was observed in PET00 and PET3.5 at temperatures of $90^{\circ} \mathrm{C}$ and $100^{\circ} \mathrm{C}$ and the fast strain rate of $0.1 / \mathrm{s}$ in the strain state of plane strain compression. This suggests that the onset of strain-induced crystallization during deformation does not necessarily depend upon the occurrence of a hold, but in all cases crystallization only significantly altered the stress-stiffening behavior at the fastest strain rate tested. 


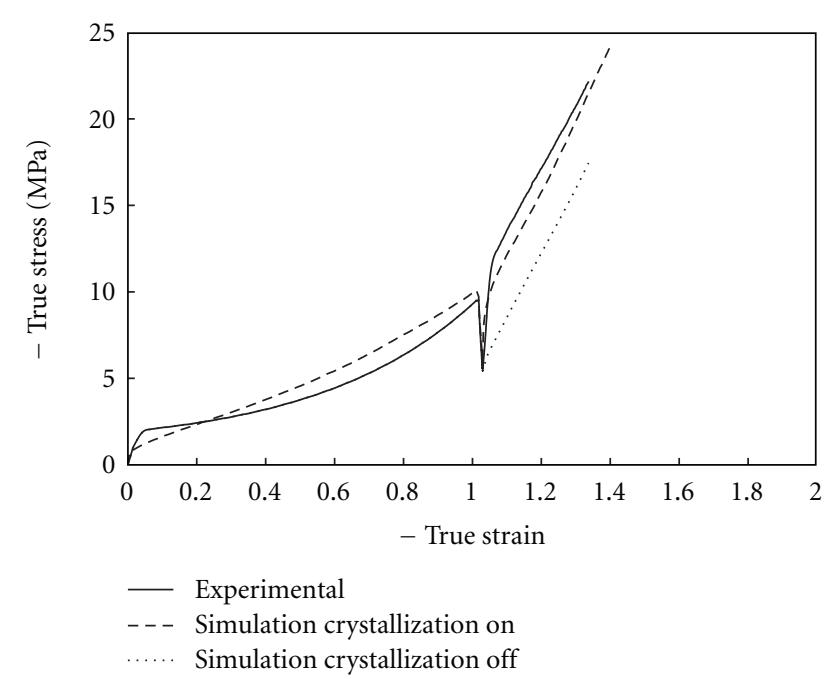

Figure 12: Comparison with experiment, PET3.5, $90^{\circ} \mathrm{C}, 0.1 / \mathrm{s}$, plane strain compression.

Based on this evidence, the conditions required to cause strain-induced crystallization during deformation in PET may be more appropriately (1) fast strain rate $(0.1 / \mathrm{s}$ and above), (2) deformation beyond a certain level $\left(\lambda_{c h}>1.55\right)$, and (3) mode of deformation that leads to a highly unidirectional molecular alignment (plane strain compression). An alternate approach would be to prescribe the chain stretch $\left(\lambda_{c h}\right)$ to change according to rate (and possibly also temperature), similar to the approach used in previous models of strain-induced crystallization in PET $[5,6]$. We leave these comparisons to future work and report here the results using our first proposed set of crystallization criteria.

\section{Simulation Results and Discussions}

To illustrate the effects of using these onset criteria in a constitutive model, the Dupaix-Krishnan [12] model was used with the proposed set of crystallization criteria.

Figure 12 shows a comparison of the experimental and simulated stress-strain curves (both with and without the crystallization part) of PET3.5 at a temperature of $90^{\circ} \mathrm{C}$, a strain rate of $-0.1 / \mathrm{s}$ in plane strain compression, and a hold period at a true strain of -1.0 . The drop in the stress level at the start of the hold condition and the subsequent strain stiffening that occurs due to crystallization is captured very well when the crystallization part is included.

\section{Conclusion and Future Work}

7.1. Conclusions. Five PET-PCT blends (PET00, PET1.5, PET3.5, PET 12, and PETG) were tested over a range of temperatures and strain rates in uniaxial and plane strain compression. Results showed that under conditions favorable to strain-induced crystallization, the low-PCT content materials (PET00, PET1.5, and PET3.5) exhibited strain hardening at high strains while the high-PCT content materials (PET12 and PETG) did not.
Load-hold tests were conducted to verify the hypothesis that the hold condition was one of the criteria that initiated strain-induced crystallization during deformation in PET. The results showed that under experimental conditions that favored crystallization, PET3.5 exhibited dramatic strain hardening while PETG did not. Three significant observations were made based on these results and they were subsequently used to prescribe the values of the crystallization initiation criteria including the chain stretch, the drop in strain rate at the hold condition, and the combination of principal stretches used to identify the strain state.

However, on comparing the monotonic test results with the load-hold test results, it was seen that the experimental conditions at which strain hardening occurs in PET00 and PET3.5 are the same in the monotonic tests and the loadhold tests. This led to the conclusion that the onset of straininduced crystallization during deformation may not depend upon the presence of a hold. The essential criteria for the occurrence of strain-induced crystallization were identified to be (1) fast strain rate $(0.1 / \mathrm{s}$ and above), (2) deformation beyond a certain level $\left(\lambda_{c h}>1.55\right)$, (3) temperature of $90^{\circ} \mathrm{C}-$ $100^{\circ} \mathrm{C}$, and (4) mode of deformation that leads to a highly unidirectional molecular alignment (plane strain compression).

\section{References}

[1] R. B. Dupaix and M. C. Boyce, "Finite strain behavior of poly(ethylene terephthalate) (PET) and poly(ethylene terephthalate)-glycol (PETG)," Polymer, vol. 46, no. 13, pp. 48274838, 2005.

[2] J. S. Zaroulis and M. C. Boyce, "Temperature, strain rate, and strain state dependence of the evolution in mechanical behaviour and structure of poly(ethylene terephthalate) with finite strain deformation," Polymer, vol. 38, no. 6, pp. 13031315, 1997.

[3] P. G. Llana and M. C. Boyce, "Finite strain behavior of poly(ethylene terephthalate) above the glass transition temperature," Polymer, vol. 40, no. 24, pp. 6729-6751, 1999.

[4] D. J. Blundell, A. Mahendrasingam, C. Martin et al., "Orientation prior to crystallisation during drawing of poly(ethylene terephthalate)," Polymer, vol. 41, no. 21, pp. 7793-7802, 2000.

[5] C. P. Buckley, D. C. Jones, and D. P. Jones, "Hot-drawing of poly(ethylene terephthalate) under biaxial stress: application of a three-dimensional glass-rubber constitutive model," Polymer, vol. 37, no. 12, pp. 2403-2414, 1996.

[6] M. C. Boyce, S. Socrate, and P. G. Llana, "Constitutive model for the finite deformation stress-strain behavior of poly(ethylene terephthalate) above the glass transition," Polymer, vol. 41, no. 6, pp. 2183-2201, 2000.

[7] M. Vigny, A. Aubert, J. M. Hiver, M. Aboulfaraj, and C. G'Sell, "Constitutive viscoplastic behavior of amorphous PET during plane-strain tensile stretching," Polymer Engineering and Science, vol. 39, no. 12, pp. 2366-2376, 1999.

[8] R. B. Dupaix and M. C. Boyce, "Constitutive modeling of the finite strain behavior of amorphous polymers in and above the glass transition," Mechanics of Materials, vol. 39, no. 1, pp. 3952, 2007.

[9] R. B. Dupaix, Temperature and rate dependant finite strain behavior of poly (ethylene terephthalate) and poly (ethylene terephthalate)-glycol above the glass transition temperature, Ph.D. thesis, MIT, 2003. 
[10] S. Ahzi, A. Makradi, R. V. Gregory, and D. D. Edie, "Modeling of deformation behavior and strain-induced crystallization in poly(ethylene terephthalate) above the glass transition temperature," Mechanics of Materials, vol. 35, no. 12, pp. 1139 $1148,2003$.

[11] A. Makradi, S. Ahzi, R. V. Gregory, and D. D. Edie, "A twophase self-consistent model for the deformation and phase transformation behavior of polymers above the glass transition temperature: application to PET," International Journal of Plasticity, vol. 21, no. 4, pp. 741-758, 2005.

[12] P. J. Flory, "Thermodynamics of crystallization in high polymers. I. Crystallization induced by stretching," Journal of Chemical Physics, vol. 15, no. 6, pp. 397-408, 1947.

[13] R. B. Dupaix and D. Krishnan, "A constitutive model for strain-induced crystallization in poly(ethylene terephthalate) (PET) during finite strain load-hold simulations," Journal of Engineering Materials and Technology, vol. 128, no. 1, pp. 2833, 2006.

[14] L. P. Chen, A. F. Yee, and E. J. Moskala, "The molecular basis for the relationship between the secondary relaxation and mechanical properties of a series of polyester copolymer glasses," Macromolecules, vol. 32, no. 18, pp. 5944-5955, 1999.

[15] E. M. Arruda and M. C. Boyce, "A three-dimensional constitutive model for the large stretch behavior of rubber elastic materials," Journal of the Mechanics and Physics of Solids, vol. 41, no. 2, pp. 389-412, 1993.

[16] A. C. Middleton, R. A. Duckett, I. M. Ward, A. Mahendrasingam, and C. Martin, "Real-time FTIR and WAXS studies of drawing behavior of poly(ethylene terephthalate) films," Journal of Applied Polymer Science, vol. 79, no. 10, pp. 1825-1837, 2001.

[17] A. Mahendrasingam, C. Martin, W. Fuller et al., "Effect of draw ratio and temperature on the strain-induced crystallization of poly (ethylene terephthalate) at fast draw rates," Polymer, vol. 40, no. 20, pp. 5553-5565, 1999.

[18] A. Mahendrasingam, D. J. Blundell, C. Martin et al., "Influence of temperature and chain orientation on the crystallization of poly(ethylene terephthalate) during fast drawing," Polymer, vol. 41, no. 21, pp. 7803-7814, 2000.

[19] A. Mahendrasingam, C. Martin, W. Fuller et al., "Observation of a transient structure prior to strain-induced crystallization in poly(ethylene terephthalate)," Polymer, vol. 41, no. 3, pp. 1217-1221, 2000. 

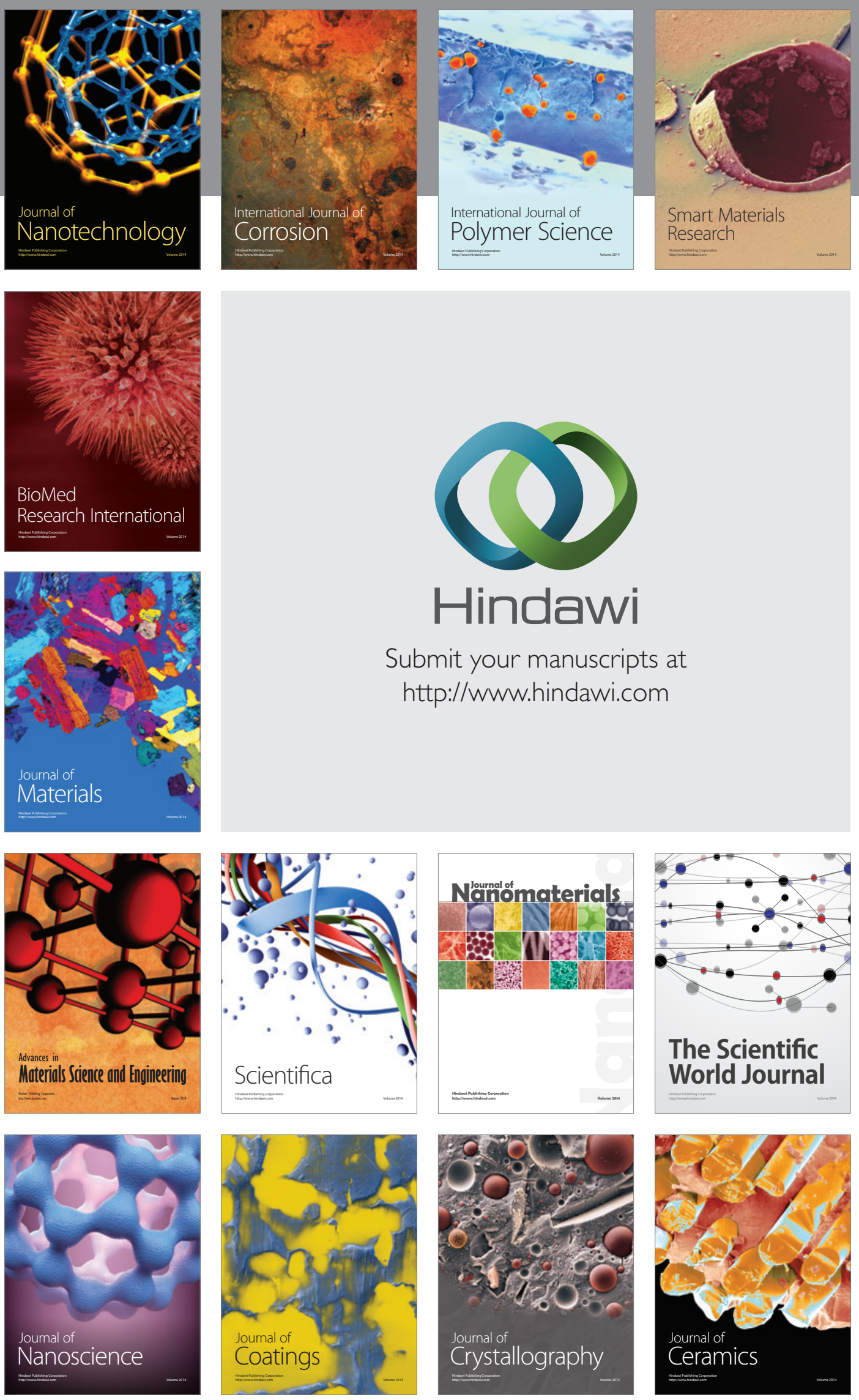

The Scientific World Journal

Submit your manuscripts at

http://www.hindawi.com

\section{World Journal}

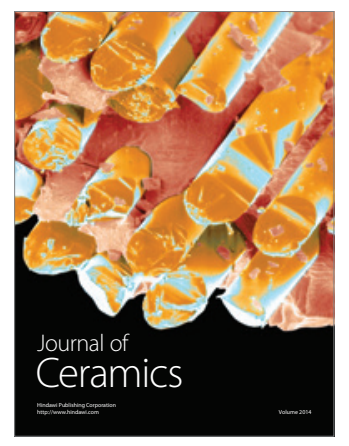

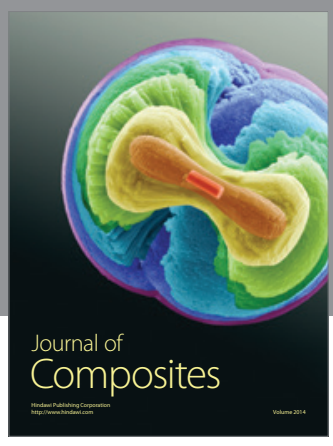
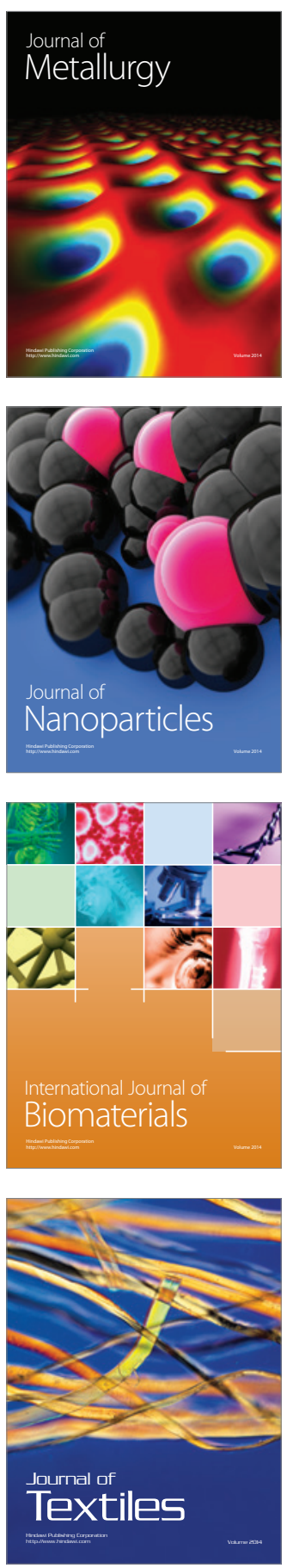